TRANSACTIONS OF THE

AMERICAN MATHEMATICAL SOCIETY

Volume 184, October 1973

\title{
WANDERING OUT TO INFINITY OF DIFFUSION PROCESSES
}

\author{
BY
}

\section{AVNER FRIEDMAN( ${ }^{(1)}$}

ABSTRACT. Let $\xi(t)$ be a diffusion process in $R^{n}$, given by $d \xi=b(\xi) d t+$ $\sigma(\xi) d w$. Conditions are given under which either $|\xi(t)| \rightarrow \infty$ as $t \rightarrow \infty$ with probability 1 , or $\xi(t)$ visits any neighborhood at a sequence of times increasing to infinity, with probability 1 . The results are obtained both in case (i) $\sigma(x)$ is nondegenerate, and (ii) $\sigma(x)$ is degenerate at a finite number of points and hypersurfaces.

Introduction。Let $w(t)=\left(w^{1}(t), \cdots, w^{n}(t)\right)$ be an $n$-dimensional Brownian motion. It is well known $[6$, p. 236] that

(i) if $n \geq 3$ then, as $t \rightarrow \infty, w(t)$ wanders out to $\infty$ in the sense that $P\{|w(t)| \rightarrow \infty$ if $t \rightarrow \infty\}=1$;

(ii) if $n=2$ then $w(t)$ visits each disc at a sequence of times increasing to $\infty$.

The purpose of this paper is to establish this behavior for general diffusion processes; more specifically, for solutions $\xi(t)$ of stochastic differential systems. For simplicity, we shall take the coefficients to be time-independent. Thus, $\xi(t)$ is the solution of an $n$-dimensional system

$$
d \xi(t)=b(\xi(t)) d t+\sigma(\xi(t)) d w(t),
$$

where $\xi(t)=\left(\xi_{1}(t), \cdots, \xi_{n}(t)\right), b(x)=\left(b_{1}(x), \cdots, b_{n}(x)\right)$, and $\sigma(x)$ is $n \times n$ matrix $\left(\sigma_{i j}(x)\right)$.

In $\S \S 1,2$ we consider the case where the matrix $\sigma(x)$ is nondegenerate for all $x \in R^{n}$. In $\S 1$, we prove a theorem of the form (i), and in $\S 2$ we prove a theorem of the form (ii). The conditions under which these theorems are proved are rather sharp. Moreover, they do not depend exclusively upon the dimension $n$. Thus, some diffusion processes wander out to $\infty$ even though $n=2$, whereas others, with $n \geq 3$, visit any ball at a sequence of time increasing to $\infty$.

In $\$ \$ 3,4$ we consider the case where the matrix $\sigma(x)$ is degenerate.

Finally, in $\$ 5$ we complement the results of $\$ 1$ by deriving an estimate on the

Received by the editors January 30, 1973. $60 \mathrm{~J} 65$.

AMS (MOS) subject classifications (1970). Primary 60H10; Secondary 60H05, 60J60,

Key words and phrases. Brownian motion, stochastic differential equations, nondegenerate diffusion, degenerate diffusion, hitting time, fundamental solution of parabolic equation. $35347 \mathrm{X}$

(1) This work was partially supported by National Science Foundation Grant GP- 
rate at which $|\xi(t)| \rightarrow \infty$ as $t \rightarrow \infty$; it is proved that

$$
P_{x}\left\{|\xi(t)| t^{\theta} \quad \infty \text { as } t-\infty\right\}=1 \text {, for any } 0<\theta<1 / 2 \text {. }
$$

1. Nondegenerate diffusion: Wandering out to infinity. Set

$$
\begin{aligned}
& a_{i j}=\frac{1}{2} \sum_{k=1}^{n} \sigma_{i k} \sigma_{j k}, \\
& L u \equiv \sum_{i, j=1}^{n} a_{i j}(x) \frac{\partial^{2} u}{\partial x_{i} \partial x_{j}}+\sum_{i=1}^{n} b_{i}(x) \frac{\partial u}{\partial x_{i}} .
\end{aligned}
$$

We shall assume

$\left(A_{1}\right)$ For all $x \in R^{n}$,

$$
\sum_{i=1}^{n}\left|b_{i}(x)\right|+\sum_{i, j=1}^{n}\left|\sigma_{i j}(x)\right| \leq C(1+|x|) \quad(C \text { constant })
$$

for any $R>0$ there is a positive constant $C_{R}$ such that

$$
\sum_{i=1}^{n}\left|b_{i}(x)-b_{i}(y)\right|+\sum_{i, j=1}^{n}\left|\sigma_{i j}(x)-\sigma_{i j}(y)\right| \leq C_{R}|x-y|
$$

if $|x|<R,|y|<R$.

$\left(\mathrm{A}_{2}\right)$ The matrix $\left(a_{i j}(x)\right)$ is positive definite for each $x \in R^{n}$.

Let

$$
\begin{gathered}
A(x, \xi)=\frac{1}{|\xi|^{2}} \sum_{i, j=1}^{n} a_{i j}(x) \xi \xi_{i}, \\
B(x)=\sum_{i=1}^{n} a_{i i}(x), \quad C(x, \xi)=\sum_{i=1}^{n} \xi_{i} b_{i}(x),
\end{gathered}
$$

and set

$$
S(x, \xi)=\frac{B(x)-A(x, \xi)+C(x, \xi)}{A(x, \xi)}, \quad S(x)=S(x, x) .
$$

We shall need the assumption:

$\left(\mathrm{A}_{3}\right)$ There is a positive constant $R_{0}$ such that

$$
S(x) \geq 1+\epsilon(|x|) \text { if }|x| \geq R_{0},
$$

where $\epsilon(r)$ is a continuous function satisfying 


$$
\int_{R_{0}}^{\infty} \frac{1}{t} \exp \left[-\int_{R_{0}}^{t} \frac{\epsilon(s)}{s} d s\right] d t<\infty .
$$

Notice that (1.3) holds for any of the functions $\epsilon(s)=d, \epsilon(s)=A / s, \epsilon(s)=B /(\log s)$ where $d, A, B$ are positive constants and $B>1$.

Theorem 1.1. Let $\left(\mathrm{A}_{1}\right)-\left(\mathrm{A}_{3}\right)$ bold. Then, for any $x \in R^{n}$,

$$
P_{x}\{|\xi(t)| \rightarrow \infty \text { if } t \rightarrow \infty\}=1 \text {. }
$$

Proof. Let $a>0$. By $\left(A_{3}\right)$, there is a continuous function $\theta(r)$ defined for $r \geq a$ such that

$$
\begin{aligned}
& S(x) \geq \theta(|x|) \quad \text { if }|x| \geq a, \\
& \theta(r)=1+\epsilon(r) \quad \text { if } r \geq R_{0} .
\end{aligned}
$$

We shall construct a function $f(x)=F(r)$, where $r=|x|$, such that $L f(x) \leq 0$ if $|x| \geq a$. As easily verified,

$$
L f(x)=A(x, x) F^{\prime \prime}(r)+\left(F^{\prime}(r) / r\right)[B(x)-A(x, x)+C(x, x)] .
$$

Hence, if

$$
\begin{gathered}
F^{\prime}(r) \leq 0 \text { for } r \geq a, \\
F^{\prime \prime}(r)+(\theta(r) / r) F^{\prime}(r)=0 \text { for } r \geq a,
\end{gathered}
$$

then, by (1.5)-(1.7),

$$
L f(x) \leq 0 \text { if }|x| \geq a .
$$

Set

$$
I(r)=\int_{a}^{r} \frac{\theta(s)}{s} d s
$$

Then a solution of (1.9) is given by

$$
F(r)=\int_{r}^{\infty} e^{-I(s)} d s
$$

the integral is convergent; by (1.3), (1.6). Notice that (1.8) is also satisfied. Hence (1.10) holds when $F$ is given by (1.12).

By Itô's formula and (1.10), if $|x|>\alpha$ then

$$
E_{x} F(|\xi(\tau)|)-E_{x} F(|x|)=E_{x} \int_{0}^{\tau} L f(\xi(s)) d s \leq 0
$$


where $r$ is any bounded stopping time such that $|\xi(s)| \geq a$ if $0 \leq s \leq \tau$. Let $\beta>\alpha$, and let $\tau_{a \beta}$ denote the exit time from the shell $\{y ; \alpha<|y|<\beta\}$. Denote by $P_{x}(\alpha)$ the probability that $\left|\xi\left(\tau_{\alpha \beta}\right)\right|=\alpha$ (given $\xi(0)=x$ ) and by $P_{x}(\beta)$ the probability that $\left|\xi\left(r_{a \beta}\right)\right|=\beta$ (given $\xi(0)=x$ ). Setting $\tau=T \wedge \tau_{a \beta}$ in (1.13) and taking $T \rightarrow \infty$, we get (since $\tau_{\alpha \beta}<\infty$ a.s.; see Theorem 1 of [3]),

$$
F(\alpha) P_{x}(\alpha)+F(\beta) P_{x}(\beta) \leq F(|x|) .
$$

Taking $\beta \rightarrow \infty$ and using the fact that $F(\beta) \rightarrow 0$ if $\beta \rightarrow \infty$, we get

$$
\lim _{\beta \rightarrow \infty} P_{x}(\alpha) \leq \frac{F(|x|)}{F(\alpha)}
$$

Introduce the balls $B_{\rho}=\{y ;|y| \leq \rho\}(0<\rho<\infty)$ and the event

$$
\Omega(\alpha)=\left\{\xi(t) \text { hits the ball } B_{\alpha} \text { for some } t \geq 0\right\} \text {. }
$$

Then we can write $(1.14)$ in the form:

$$
P_{x}(\Omega(\alpha)) \leq F(|x|) / F(\alpha)
$$

Denote by $t_{R}$ the hitting time of the boundary of the ball $B_{R}$ by $\xi(t)$. By [3, Theorem 1], if $|x|<R$ then $E_{x} t_{R}<\infty$; consequently, $P_{x}\left\{t_{R}<\infty\right\}=1$. Introduce the event

$$
\Omega^{*}(\alpha)=\left\{\xi(t) \text { hits the ball } B_{a}\right. \text { at a sequence }
$$

$$
\text { of times increasing to } \infty \text {. }
$$

Thus $\Omega^{*}(\alpha)$ is a subset of $\Omega(\alpha)$.

$$
\begin{aligned}
& \text { Let } \alpha<|x|<R \text {. Since } P_{x}\left\{t_{R}<\infty\right\}=1, \\
& P_{x}\left(\Omega^{*}(\alpha)\right)=P_{x}\left\{\xi\left(t+t_{R}\right) \text { hits } B_{\alpha} \text { at a sequence of times increasing to } \infty\right\} \text {. }
\end{aligned}
$$

Using the strong Markov property, we get

$$
\begin{aligned}
P_{x}\left(\Omega^{*}(\alpha)\right) & =E_{x} P_{\xi\left(t_{R}\right)}\left(\Omega^{*}(\alpha)\right) \\
& \leq E_{x} P_{\xi\left(t_{R}\right)}(\Omega(\alpha)) \leq E F(R) / F(\alpha)=F(R) / F(\alpha),
\end{aligned}
$$

where (1.16) has been used. Taking $R \rightarrow \infty$, we get $P_{x}\left(\Omega^{*}(\alpha)\right)=0$. This means that

$$
P_{x}\left\{\lim _{t \rightarrow \infty}|\xi(t)| \geq \alpha\right\}=1
$$


Since $\alpha$ is arbitrary, we get

$$
P_{x}\left\{{\underset{t \rightarrow \infty}{\lim }}_{t \rightarrow \infty}|\xi(t)|=\infty\right\}=1,
$$

i.e., (1.4) holds.

We shall now replace the condition $\left(A_{3}\right)$ by

$\left(A_{3}^{\prime}\right)$ As $|x| \rightarrow \infty$,

$$
\begin{gathered}
a_{i j}(x) \rightarrow a_{i j}^{0}, \\
\sum_{i=1}^{n} x_{i} b_{i}(x) \rightarrow 0,
\end{gathered}
$$

where the matrix $\left(a_{i j}^{0}\right)$ has at least three positive eigenvalues.

Theorem 1.2. Let $\left(\mathrm{A}_{1}\right),\left(\mathrm{A}_{2}\right),\left(\mathrm{A}_{3}^{\prime}\right)$ bold. Then, for any $x \in R^{n}$, the assertion (1.4) bolds.

Proof. We can perform a nonsingular transformation $x \rightarrow x^{\prime}$ in $R^{n}$ which takes $\left(a_{i j}^{0}\right)$ into $\left(a_{i j}^{*}\right)$, where $a_{i j}^{*}=0$ if $i \neq j, a_{i i}^{*}=1$ if $i=1,2,3$ and $a_{i i}^{*}=0$ or 1 if $4 \leq i \leq n$. In the new coordinates the condition $\left(A_{3}\right)$ holds with $\epsilon(s)=d$ where $d$ is any positive constant $<1$. Now apply Theorem 1.1 .

2. Nondegenerate diffusion: Visiting small neighborhoods. We shall replace the condition $\left(A_{3}\right)$ by

$\left(\mathrm{A}_{4}\right)$ For any $z \in R^{n}$ there is a positive constant $R_{z}$ such that

$$
S(x, x-z) \leq 1+\epsilon(|x-z|) \text { if }|x-z| \geq R_{z},
$$

where $\epsilon(r)$ is a continuous function satisfying

$$
\int_{R_{*}}^{\infty} \frac{1}{t} \exp \left[-\int_{R_{*}}^{t} \frac{\epsilon(s)}{s} d s\right] d t=\infty \text { for some } R_{*}>0
$$

For simplicity we have taken $\epsilon(r)$ to be independent of $z$; but the subsequent results are unaffected if $\epsilon(r)$ is allowed to depend on $z$.

Notice that the function $\epsilon(r)=1 /(\log r)$ satisfies (2.2).

Theorem 2.1. Let $\left(A_{1}\right),\left(A_{2}\right),\left(A_{4}\right)$ bold. Then, for any $x \in R^{n}$ and for any ball $B_{a}(z)=\{y ;|y-z| \leq \alpha\}, a>0$,

(2.3) $P_{x}\left\{\xi(t)\right.$ bits $B_{\alpha}(z)$ at a sequence of times increasing to $\left.\infty\right\}=1$.

Proof. We take, for simplicity, $z=0$ and write $B_{\alpha}=B_{\alpha}(0)$. We shall first construct a function $f(x)=F(r)$ for $r=|x| \geq \alpha$ such that 


$$
L f(x) \geq 0 \text { if }|x| \geq a
$$

Let $\theta(r)$ be a continuous function such that

$$
\begin{aligned}
& S(x) \leq \theta(|x|) \quad \text { if }|x| \geq a, \\
& \theta(r)=1+\epsilon(r) \quad \text { if } r \geq R_{0} .
\end{aligned}
$$

In view of (1.7), if $F(r)$ satisfies (1.8), (1.9) then (2.4) holds. With the definition (1.11), the function

$$
F(r)=-\int_{a}^{r} e^{-I(s)} d s
$$

satisfies both (1.9) and (1.8). In view of (2.6), (2.2),

$$
F(r) \rightarrow-\infty \text { if } r \rightarrow \infty \text {. }
$$

We shall now apply the first part of (1.13) to the present function $F(r)$. Making use of (2.4) and taking $r=\tau_{a \beta} \wedge T$, we get, after letting $T \rightarrow \infty$,

$$
F(\alpha) P_{x}(\alpha)+F(\beta) P_{x}(\beta)-F(|x|) \geq 0
$$

Taking $\beta \rightarrow \infty$ in (2.9) and using (2.8), we conclude that $P_{x}(\beta) \rightarrow 0$ if $\beta \rightarrow \infty$. Hence, $P_{x}(\alpha)=1-P_{x}(\beta) \rightarrow 1$ if $\beta \rightarrow \infty$. This means that

$$
P_{x}(\Omega(a))=1
$$

where $\Omega(\alpha)$ is defined in (1.15).

For any $\rho>0$, let $\partial B_{\rho}=\{y ;|y|=\rho\}$. Let $a<R_{1}<R_{2}<\cdots<R_{m}<\cdots$, $R_{m} \rightarrow \infty$ if $m \rightarrow \infty$. Introduce Markov times

$\tau_{1}=$ first time $\xi(t)$ hits $B_{a}$;

$\sigma_{1}=$ first time $>\tau_{1}$ such that $\xi(t)$ hits $\partial B_{R_{1}}$; in general,

$\tau_{m}=$ first time $>\sigma_{m-1}$ such that $\xi(t)$ hits $B_{a}$;

$\sigma_{m}=$ first time $>r_{m}$ such that $\xi(t)$ hits $\partial B_{R_{m}}$.

By $(2.10), \tau_{1}<\infty$ a.s. Hence, by the strong Markov property,

$$
P_{x}\left(\sigma_{1}<\infty\right)=P_{x}\left(\tau_{1}<\infty, \sigma_{1}<\infty\right)=E_{x} E_{\xi\left(\tau_{1}\right)} X_{\sigma_{1}-\tau_{1}<\infty}
$$

By Theorem 1 of $[3], P_{z}\left(\sigma_{1}-\tau_{1}<\infty\right)=1$ if $|z|<R_{1}$. Since $\left|\xi\left(r_{1}\right)\right|=a<R_{1}$ a.s., we get $P_{x}\left(\sigma_{1}<\infty\right)=1$. 
We now proceed by induction. Assuming that $r_{m}<\infty$ a.s., we get

$$
P_{x}\left(\sigma_{m}<\infty\right)=P_{x}\left(\tau_{m}<\infty, \sigma_{m}<\infty\right)=E_{x} E_{\xi\left(\tau_{m}\right.}, X_{\sigma_{m}-\tau_{m}<\infty}=1
$$

since $P_{z}\left(\sigma_{m}-\tau_{m}<\infty\right)=1$ if $|z|<R_{m}$ (by [3]). Next

$$
P_{x}\left(\tau_{m+1}<\infty\right)=P_{x}\left(\sigma_{m}<\infty, \tau_{m+1}<\infty\right)=E_{x} E_{\xi\left(\sigma_{m}\right)} X_{\tau_{m+1}}-\sigma_{m}<\infty=1
$$

since, by (2.10), $P_{z}\left(\tau_{m+1}-\sigma_{m}<\infty\right)=1$ for any $z \in R^{n},|z| \geq a$.

We have thus proved, by induction, that $\tau_{m}<\infty$ a.s. for all $m$.

Now, at each time $t=\tau_{m}, \xi(t)$ hits $B_{a}$. Further, since $\left|\xi\left(\sigma_{m}\right)\right|=R_{m} \rightarrow \infty$ as $m \rightarrow \infty, \lim _{m \rightarrow \infty} \sigma_{m}=\infty$; hence also $\lim _{m \rightarrow \infty}{ }^{r}{ }_{m}=\infty$. This completes the proof of (2.3) (in case $z=0$ ).

We shall now replace the condition $\left(A_{4}\right)$ by

$\left(A_{4}^{\prime}\right)$ As $|x| \rightarrow \infty$,

$$
\begin{aligned}
& a_{i j}(x)-a_{i j}^{0}=o(1 / \log |x|), \\
& \sum\left|b_{i}(x)\right|=o(1 /|x| \log |x|),
\end{aligned}
$$

and the matrix $\left(a_{i j}^{0}\right)$ has precisely two positive eigenvalues.

Theorem 2.2. Let $\left(A_{1}\right),\left(A_{2}\right),\left(A_{4}^{\prime}\right)$ bold. Then, for any $x \in R^{n}$, the assertion (2.3) bolds for any $z \in R^{n}, a>0$.

Proof. We perform an orthogonal transformation $x \rightarrow x^{\prime}$ that takes $\left(a_{i j}^{0}\right)$ into a new matrix $\left(a_{i j}^{*}\right)$ with $a_{i j}^{*}=0$ if $i \neq j$ or if $i=j \geq 3$, and $a_{i i}^{*}=1$ if $i=1,2$. In the new coordinates, the condition $\left(A_{4}\right)$ is satisfied with $\epsilon(r)=1 /(\log r)$. Now apply Theorem 2.1 .

Remark. Suppose $\left(A_{4}^{\prime}\right)$ is replaced by

$$
\begin{gathered}
a_{i j}(x) \rightarrow a_{i j}^{0} \text { as }|x| \rightarrow \infty, \\
\sum\left|b_{i}(x)\right|=0(1 /|x|) \text { as }|x| \rightarrow \infty
\end{gathered}
$$

where the matrix $\left(a_{i j}^{0}\right)$ has precisely one positive eigenvalue. Then the assertion of Theorem 2.1 remains valid, with the same proof; here $\epsilon(r)=d$ where $d$ is any positive constant $<1$.

Example. Consider the case where $n \geq 2, b_{i} \equiv 0$ and $\sigma$ is such that

$$
1 / 2 \sigma \sigma^{*}=\left(a_{i j}\right), \quad a_{i j}=\delta_{i j}+\left(g(r) / r^{2}\right) x_{i} x_{j} \quad(r=|x|)
$$


$g(r)$ is a Lipschitz continuous function vanishing near $r=0$, and

$$
\mu \leq g(r) \leq M \text { where } \mu>-1, M<\infty(\mu, M \text { constants })
$$

The eigenvalues of $\left(a_{i j}(x)\right)$ are 1 (with multiplicity $n-1$ ) and $1+g$. Hence $\left(a_{i j}(x)\right)$ is positive definite for all $x \in R^{n}$. Clearly,

$$
S(x)-1=(n-2-g(r)) /(1+g(r)) \equiv \epsilon(r) .
$$

Hence, if $g(r)$ is such that $\epsilon(r)=A /(\log r)$ for some $A>1$ (and all large $r$ ) then the assertion of Theorem 1.1 holds. If $g(r)$ is such that $f(r)=1 /(\log r)$, then $\left(A_{4}\right)$ holds with $\epsilon(s)=1 /(\log s)+C / s$ for some positive constant $C$; consequently the assertion of Theorem 2.1 holds. This example shows that the conditions $\left(A_{3}\right),\left(A_{4}\right)$ made in Theorems 1.1, 2.1 are rather sharp.

This example also shows that the behavior asserted in Theorems 1.1 and 2.1 does not depend exclusively on the dimension $n$. In fact, given any $f(r)$ which converges to 0 as $r \rightarrow \infty$, take $g=(n-2+\epsilon) /(r+\epsilon)$ (for all large $r$ ) in the above example. Then the behavior of $\xi(t)$ does not depend on $n$; if $\epsilon(r) \geq A /(\log r)$ for some $A>1$ then $\xi(t)$ wanders out to $\infty$ (i.e., (2.3) holds), whereas if $\epsilon(r) \leq 1 /(\log r)$ then $\xi(t)$ visits any neighborhood at a sequence of times increasing to $\infty$.

Meyers and Serrin [7] have introduced the conditions $\left(A_{3}\right),\left(A_{4}\right)$ in studying the existence and uniqueness of bounded solutions $u$ for the exterior Dirichlet problem. When $\left(A_{3}\right)$ holds, they prescribe $u$ at $\infty$, but when $\left(A_{4}\right)$ holds they only require that $u$ be bounded near $\infty$. The above example is taken from [7].

3. Degenerate diffusion: Wandering out to infinity. We shall now allow the matrix $\left(a_{i j}(x)\right)$ to degenerate on a compact subset of $R^{n}$. This set will consist of a finite number of points $G_{1}=\left\{z_{1}\right\}, \cdots, G_{k_{0}}=\left\{z_{k_{0}}\right\}$ and of a finite number of hypersurfaces $\partial G_{k_{0}+1}, \cdots, \partial G_{k_{0}+k_{1}}$, where $G_{k_{0}+i}$ is a closed bounded domain with boundary $\partial G_{k_{0}+i^{\bullet}}$ It is assumed that $G_{i} \cap G_{j}=\varnothing$ if $i \neq j$. Let

$$
k=k_{0}+k_{1}, \quad G=\bigcup_{i=1}^{k} G_{i}, \quad \hat{G}=R^{n} \backslash G .
$$

Denote by $\rho_{i}(x)$ the distance from $x$ to $G_{i}$ when $x \notin$ int $G_{i}$, and let

$$
\epsilon_{0}=\min _{i \neq j} \operatorname{dist}\left(G_{i^{\prime}}, G_{j}\right)
$$

We shall assume

$$
\left(\mathrm{B}_{1}\right) \partial G_{b}\left(k_{0}+1 \leq b \leq k\right) \text { is in } C^{3} \text {. If } 1 \leq b \leq k_{0} \text { then } b_{i}\left(z_{b}\right)=0, \sigma_{i j}\left(z_{b}\right)=0
$$
for $1 \leq i, j \leq n$. If $k_{0}+1 \leq b \leq k$ then 


$$
\begin{gathered}
\sum_{i, j=1}^{n} a_{i j} \nu_{i} \nu_{j}=0 \text { on } \partial G_{b}, \\
(b, \nu)+\sum_{i, j=1}^{n} a_{i j} \frac{\partial^{2} \rho_{b}}{\partial x_{i} \partial x_{j}} \geq 0 \text { on } \partial G_{b}
\end{gathered}
$$

where $\nu$ is the outward normal to $\partial G_{b}$. Finally, the matrix $\left(a_{i j}(x)\right)$ is nondegenerate for any $x \in \hat{G}$. $x \in \hat{G}$,

If $\left(A_{1}\right),\left(B_{1}\right)$ hold then, by Friedman and Pinsky [5, Theorem 1.1], for any

$$
P_{x}\{\xi(t) \text { hits } G \text { at some time } t \geq 0\}=0 \text {. }
$$

By [5], there exists a continuous function $R(x)$ in $R^{n}$, which has the following properties:

(i) $R(x)$ is in $C^{2}(\hat{G})$;

(ii) $R(x)>0$ in $\hat{G}$;

(iii) $R(x)=\rho_{b}(x)$ if $\rho_{b}(x) \leq \epsilon_{0} ; R(x)>\epsilon_{0}$ if $\min _{b} \rho_{b}(x)>\epsilon_{0}$;

(iv) $R(x)=|x|$ if $|x|$ is sufficiently large;

(v) $\Sigma_{i, j=1}^{n} a_{i j}(x) \partial^{2} R / \partial x_{i} \partial x_{j}<0$ if $x \in \hat{G}, \nabla_{x} R(x)=0$; there are precisely $k-1$ points $x$ in $\hat{G}$ with $\nabla_{x} R(x)=0$.

Let

$$
\begin{aligned}
& \mathbb{A}=\sum_{i, j=1}^{n} a_{i j}(x) \frac{\partial R}{\partial x_{i}} \frac{\partial R}{\partial x_{j}}, \\
& \mathbb{B}=\sum_{i=1}^{n} b_{i}(x) \frac{\partial R}{\partial x_{i}}+\sum_{i, j=1}^{n} a_{i j}(x) \frac{\partial^{2} R}{\partial x_{i} \partial x_{j}}, \\
& Q=(1 / R)(\mathbb{B}-\mathscr{Q} / R) .
\end{aligned}
$$

Then, if $g(x)=\Phi(R(x))$,

$$
L g(x)=\mathcal{U}^{3}\left[\Phi^{\prime \prime}(R)+\Phi^{\prime}(R) / R\right]+R Q \Phi^{\prime}(R) .
$$

Clearly

$$
S(x)=1+R^{2}(x) Q(x) / \mathscr{Q}(x) \text { if }|x| \text { is sufficiently large. }
$$

As proved in [5], if $\overline{\lim } Q(x)<0$ as $R(x) \rightarrow 0$ and as $R(x) \rightarrow \infty$, then

$$
P_{x}\left\{\min _{1 \leq b \leq k}\left[\rho_{b}(\xi(t))\right] \rightarrow 0 \text { if } t \rightarrow \infty\right\}=1 \text { if } x \in \hat{G}:
$$


Thus, in this case, $\xi(t)$ neither wanders out to $\infty$ nor visits any neighborhood in $\hat{G}$ at a sequence of times increasing to $\infty$. Hence, in order to obtain the type of behavior asserted in $\$ \S 1,2$, we shall have to change the conditions on $Q(x)$.

As will be shown, in order to generalize the results of $\$ \$ 1,2$, to the present case where $\left(B_{1}\right)$ holds, we do not need to change the conditions $\left(A_{3}\right),\left(A_{4}\right)$ near $\infty$. We only need to impose a condition on $Q(x)$ near $R(x)=0$. This condition is

$\left(B_{2}\right)$ For some $0<\delta_{0}<\epsilon_{0}$ there is a continuous function $\epsilon(r)$, defined for $0<r \leq \delta_{0}$, such that

$$
Q(x) \geq\left(\mathbb{Q}(x) / R^{2}(x)\right)_{\epsilon}(R(x)) \text { if } 0<R(x) \leq \delta_{0},
$$

and

$$
\int_{r}^{\delta} 0 \frac{1}{s} \exp \left[\int_{s}^{\delta} \frac{\epsilon(t)}{t} d t\right] d s \rightarrow \infty \text { if } r \rightarrow 0
$$

We can take, for example, $\epsilon(s)=-1 /[\log (1 / s)]$.

Remark. The condition: $\lim Q(x)<0$ as $R(x) \rightarrow 0$ is a "stability condition", meaning that $G$ attracts $\xi(t)$ near the boundary. The condition (3.4) can be interpreted as a weak "repelling condition".

Theorem 3.1. Let $\left(\mathrm{A}_{1}\right),\left(\mathrm{B}_{1}\right),\left(\mathrm{B}_{2}\right)$ and $\left(\mathrm{A}_{3}\right)$ bold. Then, for any $x \in \hat{G}$,

$$
P_{x}\{|\xi(t)| \rightarrow \infty \text { if } t \rightarrow \infty\}=1 \text {. }
$$

Let $G$ be contained in a ball $B_{R_{*}}=\left\{y ;|y|<R_{*}\right\}$.

We shall first prove a lemma.

Lemma 3.2. Let $\left(\mathrm{A}_{1}\right),\left(\mathrm{B}_{1}\right),\left(\mathrm{B}_{2}\right)$ bold and let $\beta>R_{*}$. Then, for any $x \in \hat{G} \cap B_{\beta}$

$$
P_{x}\left\{\xi(t) \text { bits the set } \partial B_{\beta} \text { for some } t \geq 0\right\}=1 \text {. }
$$

Here $B_{\beta}$ is the ball $\{y ;|y| \leq \beta\}$ and $\partial B_{\beta}$ is its boundary.

Proof. We first construct a function $g(x)=\Phi(R(x))$ for $x \in \hat{G} \cap B_{\beta}$, such that

$$
L g(x) \leq 0 \text { if } x \in \hat{G} \cap B_{\beta} .
$$

Denote by $z_{1}, \cdots, z_{k-1}$ the points in $\hat{G}$ where $\nabla_{x} R(x)=0$. By slightly modifying the proof of Lemma 2.1 in [5], we obtain a modified function $R(x)$ for which the points $z_{1}, \cdots, z_{k-1}$ lie outside the ball $B_{\beta}$. We shall work, in the present proof, with this modified function $R(x)$; it coincides with the original $R(x)$ in the $\epsilon_{0}$-neighborhood of $G$. 
We claim that there is a continuous function $\theta(r)$ satisfying

$$
1+R^{2}(x) Q(x) / \mathfrak{C}(x) \geq \theta(R(x)) \text { if } x \in \hat{G} \cap B_{\beta},
$$

$$
\theta(r)=1+\epsilon(r) \text { if } 0<r \leq \delta_{0} \cdot
$$

Indeed, since $\mathbb{Q}(x) \neq 0$ if $x \in \hat{G}, \nabla_{x} R(x) \neq 0$, the left-hand side of (3.9) is a bounded function if $x \in \hat{G} \cap B_{\beta}, \min _{b} \rho_{b}(x) \geq \delta_{0}$. Using the assumption (3.4), the existence of $\theta(r)$ [satisfying (3.9), (3.10)] follows.

Let $\Phi(r)$ be a solution of

$$
\begin{gathered}
\Phi^{\prime \prime}(r)+\theta(r) \Phi^{\prime}(r) / r=0 \quad \text { if } 0<r<r_{0}, \\
\Phi^{\prime}(r) \leq 0 \text { if } 0<r<r_{0}
\end{gathered}
$$

where $r_{0}=\max |x| \leq \beta R(x)$. Then, upon using (3.3), (3.9) we conclude that $g(x)=\Phi(R(x))$ satisfies (3.8).

A solution of (3.11), (3.12) is given by

$$
\Phi(r)=\int_{r}^{\delta_{0}} \exp \left[\int_{s}^{\delta_{0}} \frac{\theta(t)}{t} d t\right] d s
$$

In view of (3.5),

$$
\Phi(r) \rightarrow \infty \text { if } r \rightarrow 0
$$

By Itô's formula and (3.8)

$$
E_{x} \Phi(|\xi(\tau)|)-E_{x} \Phi(|x|)=E_{x} \int_{0}^{\tau} L g(\xi(s)) d s \leq 0,
$$

where $\tau$ is any bounded stopping time such that $\xi(s) \in \hat{G} \cap B_{\beta}$ if $0 \leq s \leq \tau$. Denote by $G_{\epsilon}(\epsilon>0)$ the closed $\epsilon$-neighborhood of $G$, and denote by $\sigma_{\epsilon \beta}$ the hitting time of the set $G_{\epsilon} \cup \partial B_{\beta}$. By [3, Theorem 1], $P_{x}\left(\sigma_{\epsilon \beta}<\infty\right)=1$ if $x \notin G_{\epsilon}, x \in B_{\beta}$. Denote by $P_{x}(\epsilon)$ the probability that $\xi\left(\sigma_{\epsilon \beta}\right) \in G_{\epsilon}$ (given $\xi(0)=x$ ), and by $P_{x}(\beta)$ the probability that $\xi\left(\sigma_{\epsilon \beta}\right) \in \partial B_{\beta}$ (given $\xi(0)=x$ ). Substituting $\tau=\sigma_{\epsilon \beta} \wedge T$ in (3.15), and taking $T \rightarrow \infty$, we get

$$
\Phi(\epsilon) P_{x}(\epsilon)+\Phi(\beta) P_{x}(\beta) \leq \Phi(|x|) .
$$

Taking $\epsilon \rightarrow 0$ and using (3.14), we deduce that $P_{x}(\epsilon) \rightarrow 0$ if $\epsilon \rightarrow 0$. Hence $P_{x}(\beta) \rightarrow 1$ if $\epsilon \rightarrow 0$. But this implies the assertion of the lemma.

Let $R^{*}<a<R$ and denote by $t_{R}$ the hitting time of the ball $B_{R^{*}}$ By 
Lemma 3.2, $P_{x}\left(t_{R}<\infty\right)=1$ if $x \in \hat{G}$. Hence, by the strong Markov property (cf. the proof of Theorem 1.1),

$$
P_{x}^{\prime}\left(\Omega^{*}(\alpha)\right)=E P_{\xi\left(t_{R}\right)}\left(\Omega^{*}(\alpha)\right) \leq E P_{\xi\left(t_{R}\right)}(\Omega(\alpha)),
$$

where the notation (1.15), (1.17) is used.

Now, in the domain $\left\{y ;|y|>R^{*}\right\}$ the matrix $\left(a_{i j}(y)\right)$ is nondegenerate. Since the condition $\left(A_{3}\right)$ holds, the estimate (1.14) remains valid for $x=\xi\left(t_{R}\right)$. Hence

$$
P_{x}\left(\Omega^{*}(\alpha)\right) \leq F(R) / F(\alpha) \quad(F(r) \rightarrow 0 \text { if } r \rightarrow \infty) .
$$

We can now complete the proof, as in the case of Theorem 1.1, by taking $R \rightarrow \infty$ and noting that $a$ can be arbitrarily large.

Theorem 3.3. Let $\left(\mathrm{A}_{1}\right),\left(\mathrm{B}_{1}\right),\left(\mathrm{B}_{2}\right)$ and $\left(\mathrm{A}_{3}^{\prime}\right)$ bold. Then for any $x \in \hat{G}$ the assertion (3.6) bolds.

Proof. Proceeding as in the proof of Theorem 3.1, it remains to establish the estimate (1.14). We now perform a nonsingular transformation as in the proof of Theorem 1.2.

4. Degenerate diffusion: Visiting small neighborhoods.

Theorem 4.1. Let $\left(A_{1}\right),\left(B_{1}\right),\left(B_{2}\right)$ and $\left(A_{4}\right)$ bold. Then, for any ball $B_{\alpha}(z)=$ $\{y ;|y-z| \leq \alpha\}, \alpha>0$, lying entirely in $\hat{G}$, the assertion (2.3) bolds.

Proof. For simplicity we take $z=0$. Let $R$ be any positive number such that $R>a$ and such that $G$ is contained in the interior of the ball $B_{R^{*}}$. We shall prove: if $x \in \partial B_{R}$ then

$$
P_{x}\left\{\xi(t) \text { hits the ball } B_{a} \text { for some } t \geq 0\right\}=1 \text {. }
$$

Let $\delta>0$ be sufficiently small so that $\delta<\epsilon_{0}$, the closed $\delta$-neighborhood $G_{\delta}$ of $G$ lies in the interior of $B_{R}$, and $G_{\delta} \cap B_{a}=\varnothing$.

We shall construct a function $f(x)=F(R(x))$ in $R^{n} \backslash G_{\delta}$ such that

$$
\begin{gathered}
L f(x) \geq 0 \quad \text { if } x \in R^{n} \backslash G_{\delta}, \\
F(r) \rightarrow-\infty \quad \text { if } r \rightarrow \infty .
\end{gathered}
$$

Notice that at the points $z_{m}(m=1, \cdots, k-1)$ where $\nabla_{x} R(x)=0, \mathbb{Q}=0$ and, therefore, by the property (v) of $R(x), Q<0$. Hence, $Q(x)<0$ in a neighborhood of each point $z_{m}$. It follows that there is a continuous function $\theta(r), \delta \leq r<\infty$, such that 


$$
1+R^{2} Q / \mathscr{Q} \leq \theta(R) \text { if } x \in R^{n \backslash} \backslash G_{\delta^{\circ}}
$$

In view of $\left(A_{4}\right)$, we can choose $\theta(r)$ so that, for all $r$ sufficiently large, $\theta(r)=$ $1+\epsilon(r)$ where $\epsilon(r)$ satisfies (2.2). If we now define $F(r)$, for $r \geq \delta$, by

$$
F(r)=-\int_{\delta}^{r} e^{-I(s)} d s, \quad I(s)=\int_{\delta}^{s} \frac{\theta(t)}{t} d t
$$

then $F^{\prime}(r) \leq 0$, and (4.2), (4.3) hold. Arguing as in the proof of Theorem 2.1 (following (2.8)) with the present function $f(x)=F(R(x)$ ) and with the set $\{y ;|y| \leq a\}$ replaced by $G_{\delta}$, we conclude that for any $x \in R^{n} \backslash G_{\delta}$,

$$
P_{x}\left\{\xi(t) \text { hits the set } G_{\delta} \text { for some } t \geq 0\right\}=1 \text {. }
$$

Let $\beta>R$ and denote by $\hat{G}_{\alpha \beta \delta}$ the domain bounded by $\partial G_{\delta}, \partial B_{a}, \partial B_{\beta}$. Denote by $\tau_{a \beta}$ the exit time from this domain. By [3, Theorem 1$], P_{x}\left(r_{\alpha \beta}<\infty\right)=1$ if $x \in \hat{G}_{\alpha \beta \delta}$. Using the strong Markov property we get, for any $x \in \partial B_{R}$, $P_{x}\left\{\xi(t)\right.$ hits $B_{a}$ for some $\left.t \geq 0\right\}$

$=P_{x}\left\{\xi\left(t+\tau_{\alpha \beta}\right)\right.$ hits $B_{\alpha}$ for some $\left.t \geq 0\right\}$

$=E_{x} P_{\xi\left(\tau_{\alpha \beta}\right)}\left\{\xi(t)\right.$ hits $B_{a}$ for some $\left.t \geq 0\right\}$ $\geq P_{x}\left\{\xi\left(\tau_{\alpha \beta}\right) \in \partial B_{a}\right\}+P_{x}\left\{\xi\left(\tau_{a \beta}\right) \in \partial G_{\delta}\right\} \inf _{y \in \partial G_{\delta}} P_{y}\left\{\xi(t)\right.$ hits $B_{a}$ for some $\left.t \geq 0\right\}$.

Denote by $\sigma_{\alpha R}$ the hitting time of $B_{\alpha} \cup \partial B_{R}$. By Lemma 3.2, if $y \in \partial G_{\delta}$, then $P_{y}\left(\sigma_{a R}<\infty\right)=1$. Hence, by the strong Markov property,

$$
\begin{aligned}
P_{y}\left\{\xi(t) \text { hits } B_{a} \text { for some } t \geq 0\right\} \\
=P_{y}\left\{\xi\left(t+\sigma_{a R}\right) \text { hits } B_{a} \text { for some } t \geq 0\right\} \\
=E_{y} P_{\xi\left(\sigma_{a R}\right)}\left\{\xi(t) \text { hits } B_{a} \text { for some } t \geq 0\right\} \\
=P_{y}\left\{\xi\left(\sigma_{a R}\right) \in \partial B_{a}\right\} \\
\quad+\left(1-P_{y}\left\{\xi\left(\sigma_{a R}\right) \in \partial B_{a}\right\}\right) \inf _{x \in \partial B_{R}} P_{x}\left\{\xi(t) \text { hits } B_{a} \text { for some } t \geq 0\right\} .
\end{aligned}
$$

Combining this with the previous inequality, and setting

$$
\begin{aligned}
P_{x}(\alpha) & =P_{x}\left\{\xi(t) \text { hits } B_{a} \text { for some } t \geq 0\right\}, \\
\gamma_{a \beta}(x) & =P_{x}\left\{\xi\left(\tau_{a R}\right) \in \partial B_{a}\right\} \\
\gamma_{\beta}(x) & =P_{x}\left\{\xi\left(\tau_{a R}\right) \in \partial G_{\delta}\right\} \\
\mu(y) & =P_{y}\left\{\xi\left(\sigma_{a R}\right) \in \partial B_{a}\right\}
\end{aligned}
$$


we arrive at the inequality,

$$
P_{x}(\alpha) \geq \gamma_{a \beta}(x)+\gamma_{\beta}(x) \inf _{y \in \partial G_{\delta}}\left\{\mu(y)+[1-\mu(y)] \inf _{z \in \partial B_{R}} P_{z}(\alpha)\right\} \text {. }
$$

Note that $\gamma_{a \beta}(x)$ is the solution $u(x)$ of the Dirichlet problem:

$$
\begin{aligned}
& L u=0 \text { in } \hat{G}_{a \beta \delta}, \\
& u=1 \text { on } \partial B_{a}, \\
& u=0 \text { on } \partial G_{\delta} \cup \partial B_{\beta} .
\end{aligned}
$$

Hence, by the strong maximum principle, $\gamma_{\alpha \beta}(x)$ is positive on $\partial B_{R}$. Further, $\gamma_{\alpha \beta}(x) \nearrow$ if $\beta \nearrow$. Similar assertions are true for $\gamma_{\beta}(x)$. Since $\gamma_{\alpha \beta}(x)+\gamma_{\beta}(x) \leq 1$, we conclude that

$$
\gamma_{\beta}(x) \leq \theta<1 \quad\left(x \in \partial B_{R}\right)
$$

where $\theta$ is a constant independent of $\beta$.

Notice that (4.5) implies that

$$
\gamma_{a \beta}(x)+\gamma_{\beta}(x) \rightarrow 1 \quad \text { if } \beta \rightarrow \infty \quad\left(x \in \partial B_{R}\right) .
$$

Let $P_{a}=\inf _{z \in \partial B} P_{z}(a)$. Let $\eta$ be a positive number, and choose $x_{0}$ in $\partial B_{R}$ so that $P_{a} \geq P_{x_{0}}(\alpha)-\eta$. Let $y_{0}$ be a point in $\partial G_{\delta}$ such that

$$
\inf _{y \in \partial G_{\delta}}\left\{\mu(y)+[1-\mu(y)] P_{a}\right\} \geq\left\{\mu\left(y_{0}\right)+\left[1-\mu\left(y_{0}\right)\right]\right\} P_{a}-\eta \text {. }
$$

Applying (4.6) with $x=x_{0}$, we get

$$
P_{a} \geq \gamma_{a \beta}\left(x_{0}\right)+\gamma_{\beta}\left(x_{0}\right)\left\{\mu\left(y_{0}\right)+\left[1-\mu\left(y_{0}\right)\right] P_{\alpha}\right\}-2 \eta \text {. }
$$

Hence

$$
P_{a}\left\{1-\gamma_{\beta}\left(x_{0}\right)\left[1-\mu\left(y_{0}\right)\right]\right\} \geq \gamma_{\alpha \beta}\left(x_{0}\right)+\gamma_{\beta}\left(x_{0}\right) \mu\left(y_{0}\right)-2 \eta \text {. }
$$

Taking $\beta$ sufficiently large and using (4.8) with $x=x_{0}$, we get

$$
P_{a}\left\{1-\gamma_{\beta}\left(x_{0}\right)\left[1-\mu\left(y_{0}\right)\right]\right\} \geq\left\{1-\gamma_{\beta}\left(x_{0}\right)\left[1-\mu\left(y_{0}\right)\right]\right\}-3 \eta \text {. }
$$

Denote the expression in braces by $\lambda_{\beta}$. From (4.7) it follows that $\lambda_{\beta} \geq$ $1-\theta>0$. Hence

$$
P_{a} \geq 1-3 \eta / \lambda_{\beta} \geq 1-3 \eta /(1-\theta)
$$


Since $\eta$ is arbitrary, $P_{\alpha}=1$. This implies that $P_{\alpha}(x)=1$ for all $x \in \partial G_{R}$, i.e., (4.1) holds.

Having proved (4.1), we can now easily complete the proof of Theorem 4.1 by the argument given in the proof of Theorem 2.1 (from (2.10) on). Instead of (2.10) we use (4.1), and instead of Theorem 1 of [3] we use Lemma 3.2.

Remark 1. Theorem 4.1 remains true if the condition $\left(A_{4}\right)$ is replaced by $\left(A_{4}^{\prime}\right)$, or by the conditions in the remark following Theorem 2.2 .

Remark 2. When the $\sigma_{i j}(x)$ are linear homogeneous functions, the matrix $\left(a_{i j}(x)\right)$ degenerates at the origin and (possibly) along rays initiating at the origin. In case the $b_{i}(x)$ are also linear homogeneous functions the behavior of $\xi(t)$ was studied in detail in [4]. The function $Q(x)$ is now homogeneous. If $Q(y)<0$ for all $y,|y|=1$, then $P_{x}\{\xi(t) \rightarrow 0$ if $t \rightarrow \infty\}=1$ for all $x$. If $Q(y)>0$ for all $y,|y|=1$, then $P_{x}\{|\xi(t)| \rightarrow \infty$ if $t \rightarrow \infty\}=1$ for all $x \neq 0$.

5. The rate of wandering out to infinity. In this section we return to the situation of Theorem 1.1. We shall assume

$\left(A_{s}\right) a_{i j}(x), b_{i}(x)$ are bounded functions in $R^{n}$, the $a_{i j}(x)$ are uniformly Hölder continuous in $R^{n}$, and, for all $x \in R^{n}, \xi \in R^{n}$,

$$
\sum_{i, j=1}^{n} a_{i j}(x) \xi_{i} \xi_{j} \geq a_{0}|\xi|^{2} \quad\left(a_{0} \text { positive constant }\right) .
$$

We shall also assume that the function $\epsilon(r)$ occurring in the condition $\left(A_{3}\right)$ satisfies, for some $r_{0}$ sufficiently large,

$$
\epsilon(r)=d \quad \text { if } r \geq r_{0} \quad(d \text { positive constant }) .
$$

Theorem 5.1. Let $\left(A_{1}\right),\left(A_{5}\right)$ bold and let $\left(A_{3}\right)$ bold with $\epsilon(r)$ satisfying (5.1). Then, for any $0<\theta<1 / 2, x \in R^{n}$,

$$
P_{x}\left\{|\xi(t)| / t^{\theta} \rightarrow \infty \text { if } t \rightarrow \infty\right\}=1 .
$$

For $\xi(t)$ a Brownian motion $w(t)$, this result was proved by Dvoretsky and Erdös [1]. As in [1], the function $t^{\theta}$ occurring in (5.2) can actually be replaced by $t^{1 / 2} g(t) \lambda(t)$ where $g(t), \lambda(t)$ are positive, monotone decreasing, $\lambda(t) \rightarrow 0$ if $t \rightarrow \infty$, and

$$
\sum_{n=1}^{\infty}\left[g\left(2^{m}\right)\right]^{d}{ }^{0}<\infty \text { for some } d_{0} \leq d, d_{0}<n
$$

More recently, Theorem 5.1, under more restrictive assumptions, was proved by Friedman [3]. The proof in [3] exploits the result of Dvoretsky-Erdös together 
with estimates on $E_{x}\left|\xi(t)-\sigma_{0} w(t)\right|^{2}$, where $\sigma_{0}=\lim \sigma(x)$ as $|x| \rightarrow \infty$. The present proof is entirely different from that in [3]; it is modeled after the proof in [1] for $\xi(t)=w(t)$.

We shall first prove two lemmas.

Lemma 5.2. Let $\left(A_{1}\right),\left(A_{5}\right)$ and $\left(A_{3}\right),(5.1)$ bold. Then there exists a positive constant $C$ such that for any $a>r_{0}, x \in R^{n}$,

$$
P_{x}\{|\xi(t)| \leq a \text { for some } t \geq 0\} \leq C(\alpha /|x|)^{d} \text {. }
$$

Proof. Let $R>a$. Consider the Dirichlet problem:

$$
\begin{aligned}
L u_{R}(x)=0 & \text { if } \alpha<|x|<R, \\
u_{R}=1 & \text { if }|x|=\alpha, u_{R}=0 \text { if }|x|=R .
\end{aligned}
$$

Let $f(x)=F(r)(r=|x|)$ be the function constructed in the proof of Theorem 1.1. Then $v(x)=F(r) / F(\alpha)(r=|x|)$ satisfies

$$
\begin{aligned}
L v & \leq 0 \quad \text { if } a<|x|<R, \\
v & =1 \quad \text { if }|x|=a, \quad v \geq 0, \quad \text { if }|x|=R .
\end{aligned}
$$

Hence, by the maximum principle,

$$
0 \leq u_{R}(x) \leq v(x)
$$

By a standard argument (cf. [7]), there exists a sequence $R=R_{m} \nearrow \infty$ for which the $u_{R}(x)$ converge point-wise to a solution $u$ of

$$
L u=0 \quad \text { if }|x|>a, \quad u=1 \quad \text { if }|x|=1 .
$$

From (5.4) we get

$$
0 \leq u(x) \leq v(x)=\frac{\int_{r}^{\infty} \exp [-I(s)] d s}{\int_{a}^{\infty} \exp [-I(s)] d s} .
$$

Using (5.1) to estimate the right-hand side, we obtain,

$$
0 \leq u(x) \leq C a^{d} / r^{d}
$$

where $C$ is a constant independent of $\alpha, x$.

Now, by Ito's formula, if $\alpha<|x|<R$,

$$
u_{R}(x)=P_{x}\left\{\xi(t) \text { hits } \partial B_{a} \text { before it hits } \partial B_{R}\right\} \text {. }
$$

Hence, if $|x|>a$, 


$$
u(x)=P_{x}\left\{\xi(t) \text { hits } \partial B_{a} \text { for some } t \geq 0\right\} .
$$

Now, if $|x| \leq a$ then the assertion (5.3) is trivially true (with $C=1$ ). If, on the other hand, $|x|>a$ then the assertion (5.3) follows from (5.7), (5.6).

Lemma 5.3. Let $\left(A_{1}\right),\left(A_{5}\right)$ and $\left(A_{3}\right),(5.1)$ bold, with $d<n$. Then there is a positive constant $C^{\prime}$ such that, if $a>r_{0},|x|<\alpha / 4, T>0$,

$$
P_{x}\{|\xi(t)| \leq a \text { for some } t \geq T\} \leq C^{\prime}\left(\alpha / T^{1 / 2}\right)^{d} \text {. }
$$

Proof. By the Markov property and Lemma 5.2,

$$
\begin{aligned}
P_{x}\{|\xi(t)| \leq \alpha & \text { for some } t \geq T\} \\
& =E_{x} P_{\xi(T)}\{|\xi(t)| \leq \alpha \text { for some } t \geq 0\} \\
& =P_{x}\{|\xi(T)| \leq \alpha\}+E_{x}\{X|\xi(T)|>a \\
& \equiv I+J .
\end{aligned}
$$

Denote by $K(t, x, y)$ the fundamental solution of the parabolic operator $\partial / \partial t-L$. By [2],

$$
0 \leq K(t, x, y) \leq\left(M / t^{n / 2}\right) \exp \left[-\mu|x-y|^{2} / t\right]
$$

where $M, \mu$ are positive constants.

We can write

$$
\begin{aligned}
& I=\int_{|y| \leq a} K(T, x, y) d y, \\
& J=C a^{d} \int_{|y|>a} \frac{1}{|y|^{d}} K(T, x, y) d y .
\end{aligned}
$$

We shall subsequently denote various positive constants by the same symbol $C$. Substituting $|y-x|=\rho \sqrt{ } T$ in the integral $I$ and noting that

$$
\rho \sqrt{ } T=|y-x| \leq 2 \alpha \quad(\text { since }|y| \leq \alpha,|x|<\alpha),
$$

we get

$$
I \leq C \int_{\rho \sqrt{ } T \leq 2 a} \rho^{\rho^{n-1} e^{-\mu \rho^{2}}} d \rho \leq C\left(\frac{a}{\sqrt{T}}\right)^{n}
$$

Substituting $|y-x|=\rho \sqrt{ } T$ in the integral $J$ and noting that

$$
\begin{aligned}
& \rho \sqrt{ } T=|y-x| \geq a / 2 \quad\left(\text { since }|y| \geq a_{,}|x| \leq \alpha / 2\right), \\
& |y| \geq|y-x|-|x| \geq \rho \sqrt{ } T / 2 \quad(\text { since }|x| \leq \alpha / 4 \leq|y-x| / 2),
\end{aligned}
$$

we get 


$$
\begin{aligned}
J & \leq C \alpha^{d} \int_{\rho \sqrt{ } T>\alpha / 2} \frac{\rho^{n-1}}{(\rho \sqrt{ } T)^{d}} e^{-\mu \rho^{2}} d \rho \\
& \leq C\left(\frac{a}{\sqrt{T}}\right)^{d} \int_{\rho>0} \rho^{n-1-d} e^{-\mu \rho^{2}} d \rho \leq C\left(\frac{a}{\sqrt{ } T}\right)^{d}
\end{aligned}
$$

since $n-1-d>-1$. Substituting the estimates for $I, J$ into (5.9), the assertion (5.8) follows.

Proof of Theorem 5.1. Without loss of generality we may assume that $d<n$. We apply Lemma 5.3 with $T=2^{m}, a=2^{(m+1) \theta}$ where $m$ is a positive integer such that $|x|<2^{(m+1) \theta} / 4$. We get

$$
\begin{aligned}
P_{x}\left\{|\xi(t)| \leq t^{\theta}\right. & \text { for some } \left.t, 2^{m} \leq t \leq 2^{m+1}\right\} \\
& \leq P_{x}\left\{|\xi(t)| \leq 2^{(m+1) \theta} \text { for some } t \geq 2^{m}\right\} \\
& \leq C\left[2^{(m+1) \theta} / 2^{m / 2}\right]^{d} \leq C 2^{m(\theta-1 / 2) d} .
\end{aligned}
$$

Since $\Sigma 2^{m(\theta-1 / 2) d}<\infty$, the Borel-Cantelli lemma implies that, with probability 1 , the sequence of events

$$
\left\{|\xi(t)| \leq t^{\theta} \text { for some } t, 2^{m} \leq t \leq 2^{m+1}\right\}
$$

(where $\xi(0)=x$ ) occurs only finitely often. Hence

$$
P_{x}\left\{|\xi(t)|>t^{\theta} \text { for all } t \text { sufficiently large }\right\}=1 \text {. }
$$

Since this is true also for $\theta$ replaced by any $\theta^{\prime}, \theta<\theta^{\prime}<1 / 2$, the assertion of the theorem follows.

Corollary 5.4. Let $\left(\mathrm{A}_{1}\right),\left(\mathrm{A}_{5}\right)$ and $\left(\mathrm{A}_{3}^{\prime}\right)$ bold. Then, for any $0<\theta<1 / 2$, $x \in R^{n}$, the assertion (5.2) bolds.

Indeed, perform a nonsingular transformation as in the proof of Theorem 1.2. In the new coordinates the conditions $\left(A_{3}\right),(5.1)$ hold.

Remark. Consider the example at the end of $\$ 2$. If $n=2$ and $g=$ $-1 /(1+1 / d)(d>0)$, then the assertion of Theorem 5.1 holds. Thus, even when $n=2$, a diffusion process $\xi(t)$ may wander out to $\infty$ at a rate $\geq t$, for any $0<\theta<1 / 2$.

\section{REFERENCES}

1. A. Dvoretsky and P. Erdös, Some problems on random walk in space, Proc. Second Berkeley Sympos. on Math. Statist. and Probability (1950), Univ. of California Press, Berkeley, Calif., 1951, pp. 353-367. MR 13, 852. 
2. A. Friedman, Partial differential equations of parabolic type, Prentice-Hall, Englewood Cliffs, N. J., 1964. MR 31 \#6062.

3. - Limit behavior of solutions of stochastic differential equations, Trans. Amer. Math. Soc. 170 (1972), 359-384.

4. A. Friedman and M. A. Pinsky, Asymptotic behavior of solutions of linear stochastic differential systems, Trans. Amer. Math. Soc. 181 (1973), 1-22.

5. - Asymptotic stability and spiraling properties of solutions of stochastic equations, Trans. Amer. Math. Soc. (to appear).

6. K. Itô and H. P. McKean, Diffusion processes and their sample paths, Die Grundlehren der math. Wissenschaften, Band 125, Academic Press, New York; SpringerVerlag, Berlin, 1965. MR 33 \#8031.

7. N. Meyers and J. Serrin, The exterior Dirichlet problem for second order elliptic differential equations, J. Math. Mech. 9 (1960), 513-538. MR 22 \#8200.

DEPARTMENT OF MATHEMATICS, NORTHWESTERN UNIVERSITY, EVANSTON, ILLINOIS 60201 